INPLASY

PROTOCOL

To cite: Zhang et al. Clinical efficacy and safety of acupuncture for the treatment of drug withdrawal syndrome:

A systematic review and metaanalysis protocol. Inplasy protocol 2021100022. doi: 10.37766/inplasy2021.10.0022

Received: 07 October 2021

Published: 07 October 2021

Corresponding author:

Xiong Zhang

569094685@qq.com

Author Affiliation:

Guizhou University of

Traditional Chinese Medicine.

Support: QKHJC-ZH[2021].

Review Stage at time of this submission: The review has not yet started.

Conflicts of interest:

None declared.

\section{Clinical efficacy and safety of acupuncture for the treatment of drug withdrawal syndrome: A systematic review and meta-analysis protocol}

Zhang, X1'; Zhang, S2; Xue, K³; Zhao, X4; Che, H5; Liang, G6; Li, J7.

Review question / Objective: This study is designed so as to conduct a systematic evaluation of the effectiveness and safety of acupuncture therapy for the treatment of DWS, in addition to providing up-to-date information for using acupuncture therapy for the treatment of DWS.

Condition being studied: Drug withdrawal syndrome (DWS). Information sources: Literature databases: PubMed, Web of Science, Embase, Cochrane library, CNKI (Chinese National Knowledge Infrastructure), VIP(Chinese Scientifific Journals Database), СВM(Chinese Biomedical Literature Database) and Wanfang Databases. Trial registry: Clinicaltrials.gov, and Chinese Clinical Trial Registry. Source of grey literature: reference lists of relevant reviews.

INPLASY registration number: This protocol was registered with the International Platform of Registered Systematic Review and Meta-Analysis Protocols (INPLASY) on 07 October 2021 and was last updated on 07 October 2021 (registration number INPLASY2021100022).

\section{INTRODUCTION}

Review question / Objective: This study is designed so as to conduct a systematic evaluation of the effectiveness and safety of acupuncture therapy for the treatment of DWS, in addition to providing up-to-date information for using acupuncture therapy for the treatment of DWS.
Condition being studied: Drug withdrawal syndrome(DWS).

\section{METHODS}

Participant or population: Patients with DWS. 
Intervention: In this study, acupuncture is the intervention of interest, and it includes body acupuncture, scalp acupuncture, and/ or electric acupuncture. We will include the following two types of experimental designs: 1) acupuncture provided to the experimental group and other therapies provided to the control group and 2) acupuncture combined with other therapies provided to the experimental group and other similar therapies provided to the control group. Other therapies include the following: external treatments as part of TCM, such as moxibustion, massage, acupoint application, and ear acupoints; medications such as TCM decoctions, Chinese patent medicines, and western medicine; and cognitive behavioral therapy, placebos, and other similar therapies.

Comparator: In this study, acupuncture is the intervention of interest, and it includes body acupuncture, scalp acupuncture, and/ or electric acupuncture. We will include the following two types of experimental designs: 1) acupuncture provided to the experimental group and other therapies provided to the control group and 2) acupuncture combined with other therapies provided to the experimental group and other similar therapies provided to the control group. Other therapies include the following: external treatments as part of TCM, such as moxibustion, massage, acupoint application, and ear acupoints; medications such as TCM decoctions, Chinese patent medicines, and western medicine; and cognitive behavioral therapy, placebos, and other similar therapies.

Study designs to be included: Randomized controlled trials.

Eligibility criteria: We will refer to the Diagnostic and Statistical Manual of Mental Disorders, published by the American Psychiatric Association[28] (DSM-IV or DSM-III-R), for the inclusion of patients according to the diagnostic criteria for drug dependence, without restrictions on gender, age, course of disease or severity. The cases will all be selected from abstainers who voluntarily accepted detoxification.

Information sources: Literature databases: PubMed, Web of Science, Embase, Cochrane library, CNKI (Chinese National Knowledge Infrastructure), VIP(Chinese Scientifific Journals Database), CBM(Chinese Biomedical Literature Database) and Wanfang Databases. Trial registry: Clinicaltrials.gov, and Chinese Clinical Trial Registry. Source of grey literature: reference lists of relevant reviews.

Main outcome(s): The primary outcome will be the withdrawal symptom score, as rated according to the Himmelsbach withdrawal symptom scoring system.

Additional outcome(s): The secondary outcome will include Hamilton Depression Scale(HAMD), Hamilton anxiety scale(HARS), Pittsburgh sleep quality index(PSQI), Bristol Stool Form Scale(BSFS), Patient Assessment of Constipation sympton (PAC-SYM), Short Musculoskeletal Function Assessment (SMFA), Visual Analogue Scale(VAS), serum interleukin 2, serum immunoglobulin (IgG、

IgM和IgA), cure rate, and adverse reactions.

Quality assessment / Risk of bias analysis: The Cochrane Manual (5.3.0) RCT risk assessment tool will be used to evaluate randomization, allocation of hidden protocols, patient and doctor blindness to the treatment protocols, assessment of blinding methods, completeness of results data, selective reporting of results, and the existence of other biases. The RCT methods, as described in the papers, will be used to objectively identify each of the evaluation criteria as high risk, low risk, or unclear risk, for which the reasons will be briefly explained. Any discrepancies in the bias risk evaluation will be settled via a discussion between the two reviewers or based on the judgment of a third reviewer.

Strategy of data synthesis: RevMan $\mathbf{5 . 3}$ software will be used for statistical 
analysis(Nordic Cochrane Center, Cochrane Collaborative Organization, Copenhagen, Denmark). We will use the mean difference(MD) or standardized mean difference(SMD) to analyze continuous data and odds ratios(ORs) to analyze dichotomous data. Both types of test statistics will be expressed with a $95 \%$ confidence interval.

Subgroup analysis: If there is significant heterogeneity in the results analysis and a sufficient number of RCTs are available, we will carry out a subgroup analysis to further explore the sources of heterogeneity via two methods: 1) intervention methods: RCTs that used acupuncture will be compared with RCTs that used acupuncture combined with other nonacupuncture therapies, among which the latter is expected to have an increased effect and 2) intervention time: RCTs with an intervention time $>2$ weeks will be compared with those $\leq 2$ weeks, among which the former is expected to be more effective.

Sensitivity analysis: To determine the robustness and reliability of the metaanalysis results, we will conduct a sensitivity analysis in accordance with the criteria for sample size, heterogeneity, and statistical models; we will exclude lowquality studies.

\section{Country(ies) involved: China.}

Keywords: acupuncture, drug withdrawal syndrome, protocol, systematic review, meta-analysis.

Contributions of each author:

Author 1 - Xiong Zhang.

Email: 569094685@qq.com

Author 2 - Shengjie Zhang.

Email: 547196121@qq.com

Author 3 - Kaiyang Xue.

Email: 2317649912@qq.com

Author 4 - Xiaorui Zhao.

Email: 380151013@qq.com

Author 5 - Hongxia Che.

Email: 1252165358@qq.com
Author 6 - Guangping Liang.

Email: 2265250474@qq.com

Author 7 - Jia Li.

Email: ljgzzyydx@163.com 